\title{
A Fast CU Division Algorithm for Intra Prediction in HEVC
}

\author{
Ryota Takagi ${ }^{\mathrm{a}, *}$, Genki Yoshitake ${ }^{\mathrm{b}}$, Yankang Wang ${ }^{\mathrm{a}}$ \\ ${ }^{a}$ Department of Computer and Information Systems \\ National Institute of Technology, Kurume College \\ Fukuoka 830-8555, Japan \\ ${ }^{\mathrm{b}}$ Department of Electrical Engineering and Computer Science \\ Faculty of Engineering, Kyushu University \\ Fukuoka 819-0395, Japan \\ *ykwang@kurume-nct.ac.jp
}

\begin{abstract}
High Efficiency Video Coding (HEVC) is a new video coding standard following H.264/AVC. By introducing a flexible coding unit (CU), which can be recursively divided from $64 \times 64$ to $8 \times 8$ blocks in a Quadtree-Structure, HEVC achieves significantly higher coding efficiency than the previous standards. With the flexible CU structure, HEVC can effectively adapt to complicated contents with a smaller $\mathrm{CU}$ or to flat contents with a larger $\mathrm{CU}$, making it suitable for applications from mobile video to super high definition television (HDTV). On the other hand, CU division does incur high computational cost for HEVC. In this paper, we propose a simple and fast $\mathrm{CU}$ division algorithm by using only a subset of pixels to determine when $\mathrm{CU}$ division happens. Experiment results show that our algorithm can achieve prediction quality close to HEVC Test Model (HM) with much lower computational cost.
\end{abstract}

Keywords: HEVC, Intra Prediction, Quadtree-Structure, CU Division

\section{Introduction}

The High Efficiency Video Coding (HEVC) is the most recent video coding standard after H.264/AVC. Similar to MPEG-2/4 and H.264/AVC, HEVC uses a hybrid coding method based on motion-compensated prediction and DCT coding on blocks. In HEVC, however, because of the introduction of advanced techniques such as block division structure and intra prediction mode, compression rate is about doubled compared to H.264/AVC[1]-[5].
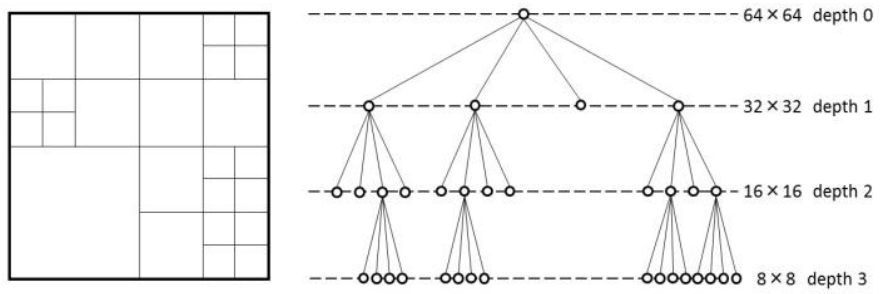

Fig. 1 CU division and Quadtree-Structure

For intra and inter predictions HEVC uses a variable block division scheme that allows a block to be divided into a set of sub-blocks of different sizes. Such block division scheme consists of 4 units: Coding Tree Unit (CTU), Coding Unit (CU), Prediction Unit (PU), and Transform Unit (TU). A picture to be coded is first divided into a number of CTUs of $64 \times 64$ pixel size, and then each CTU is divided into smaller CUs of variable sizes but no smaller than $8 \times 8$ pixels. The CU division process follows so-called Quadtree-Structure and can be recursively repeated.

Fig. 1 shows an example of recursive division of a $\mathrm{CU}$ in Z scan. Initially a CU has the same size of $64 \times 64$ as the CTU at depth 0 . When the $\mathrm{CU}$ is divided, it will be split into four sub-CUs of $32 \times 32$ pixels at depth 1 . The division process can repeat until depth 3 when the divided CUs reach the minimal size of $8 \times 8$ pixels. When division is finished on a $\mathrm{CU}, \mathrm{PU}$ is run on the eight possible partitions for inter coding and the two possible partitions for intra coding. Such $\mathrm{CU}$ division scheme is what gives HEVC the advantage in coding efficiency over the previous standards.

In HEVC, the optimal CU can be estimated using the Ratio-Distortion (RD) cost computation defined as follows, 


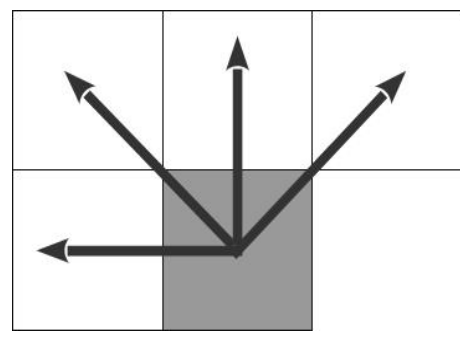

Fig. 2 Pixel search direction

$$
J_{R D}=S S E+\lambda \times R
$$

where SSE denotes the sum of squared errors, $\lambda$ is a coefficient dependent on the quantization parameter (QP), and $R$ is the total bits for encoding. In the HEVC Test Model (HM), which is the standard implementation of HEVC, all CU divisions from depth 0 to depth 3 and all PU partitions require $\mathrm{RD}$ to be computed, resulting in a large number of calculations and a significant complexity for HEVC. To reduce such complexity several algorithms have been proposed that target the reduction of RD calculation and $\mathrm{CU}$ division [6]-[10].

In this paper, we propose a simple and fast $\mathrm{CU}$ division algorithm in which only a few pixels around a CU being processed are analyzed instead of the RD calculation to decide whether the $\mathrm{CU}$ is to be divided. If there is a large variability among those pixels the $\mathrm{CU}$ will be divided; otherwise division stops.

\section{Fast Coding-Unit Division}

\subsection{Algorithm}

In the proposed method, the variability of pixel intensities around the current $\mathrm{CU}$ is estimated to decide if the CU should be divided. Such variability indicates the degree of complexity of a CU due to contents and/or motion. A CU will only be divided when the variability is high. To minimize computation when estimating the variability, we propose to check only a limited number of pixels around the $\mathrm{CU}$ instead of checking all of the pixels.

Fig. 2 shows four search directions from the current CU (gray block). In each search direction only pixels within 64 pixels plus half the size of $\mathrm{CU}$ from the center are checked. For example, if the current $\mathrm{CU}$ is of $32 \times 32$ pixels, then 80 $(64+32 / 2)$ pixels will be checked. Searching all four directions will thus require $320(4 \times 80)$ pixels to be checked. Our algorithm for CU division is therefore as follows.
Algorithm

Step1. Get a CTU from image as an initial CU of $64 \times 64$ pixels, set depth $=0$.

Step2. While depth $<3$ repeat Step3 to Step6.

Step3. Find an unprocessed CU in the current depth.

Step4. For this CU get the brightness value of the pixels in four search directions and calculate their variances.

Step5. For the four variance values, count those greater than a pre-decided threshold $T$ and record the count in $\mathrm{N}$.

Step6. If $\mathrm{N} \geq 2$, the current $\mathrm{CU}$ is divided into four sub-CUs, depth $=$ depth +1 , go back to Step 2 .

Step7. If depth $=0$ go to Step 9 .

Step8. If depth $=3$ or all the CUs in current depth have been processed depth $=$ depth -1 , go back to Step 2 .

Step9. The sizes of all CUs have been determined.

Step10. Return.

\subsection{Determination of Thresholds}

In our algorithm there can be at most 4 search steps (03 ), and in only 3 of them (0-2) is the decision needed on whether to further divide a CU. We allow three different thresholds $\left(T_{0}-T_{2}\right)$ to be used in depths 0 to 2 , respectively, and they are empirically determined using two types of standard test images, People and Landscape. Coding performance is evaluated with PSNR and coding time. PSNR is calculated as follows,

$$
P S N R=10 \log _{10} \frac{M A X V A L^{2}}{M S E}
$$

where MAXVAL is the maximal pixel value of original image, and $M S E$ is the mean square error calculated between the original image and the coded image.

Fig. 3 shows how PSNR and coding time change in depth 0 when we vary threshold $T_{0}$ from 0 to 1000 in step 100 . When $T_{0}$ is increased from 0 to around 300, PSNR either continuously decreases (People) or stays relatively stable (Landscape). When $T_{0}$ goes above 300 , PSNR may change in different directions for the two types of sequences. Coding time, on the other hand, decreases with increasing threshold over the entire range for both sequences. Balancing both PSNR and coding time, we consider threshold $T_{0}=300$ acceptable for depth 0 .

Similarly, thresholds $T_{1}$ for depth 1 and $T_{2}$ for depth 2 are selected to be 800 and 2800 , respectively. 


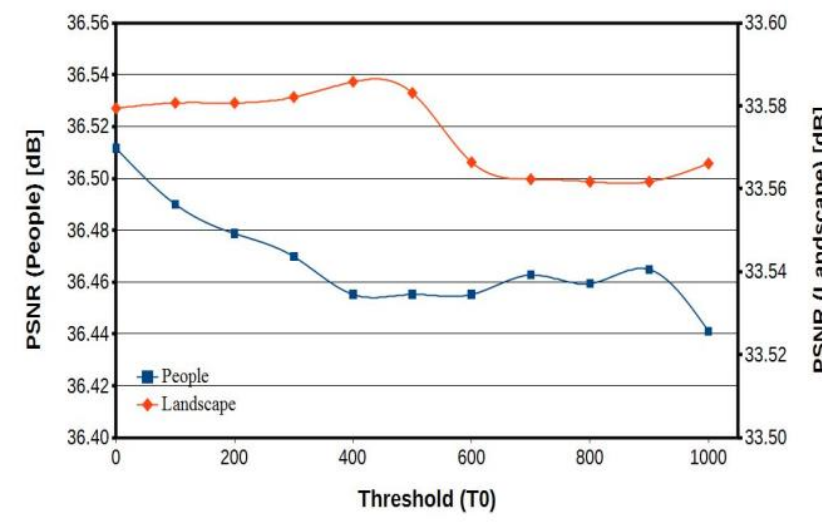

(a) Effect of $T_{0}$ on PSNR

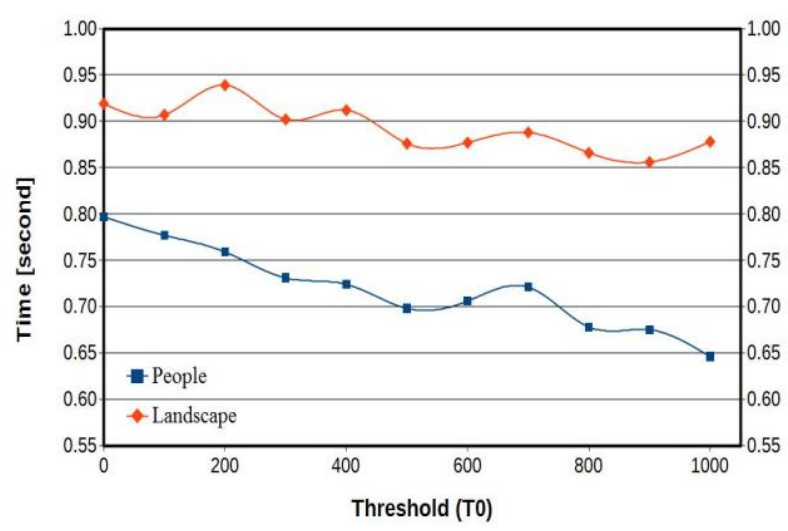

(b) Effect of $T_{0}$ on processing time

Fig. 3 Determination of threshold $T_{0}$ based on two test images

\section{Simulation Results}

In simulation experiments our algorithm is tested with PSNR and coding time for 6 standard test images in comparison with HM-16.7, where QP is set to 22, 27, 32 and 37 for both methods.

Table 1 and Table 2 show the coding time and PSNR, respectively, for HM, while Table 3 and Table 4 show the values for our algorithm. Comparing Table 1 with Table 3, our proposed algorithm is much faster than HM. On the other hand, Table 2 and Table 4 show that PSNR of our algorithm is slightly lower than that of HM.

Table 1. Coding time of HM (Sec.)

\begin{tabular}{c||c|c|c|c}
\hline Image & QP:22 & QP:27 & QP:32 & QP:37 \\
\hline \hline Lenna & 4.123 & 3.452 & 2.953 & 2.656 \\
Mandrill & 5.573 & 4.970 & 4.192 & 3.562 \\
House & 4.275 & 3.470 & 3.244 & 2.926 \\
Peppers & 4.711 & 3.662 & 3.115 & 2.736 \\
Sailboat & 5.034 & 4.268 & 3.482 & 3.066 \\
Splash & 3.766 & 3.078 & 2.713 & 2.495 \\
\hline \hline Average & 4.580 & 3.817 & 3.283 & 2.907 \\
\hline
\end{tabular}

Table 2. PSNR of HM (dB)

\begin{tabular}{c||c|c|c|c}
\hline Image & QP:22 & QP:27 & QP:32 & QP:37 \\
\hline \hline Lenna & 41.873 & 39.042 & 36.702 & 34.396 \\
Mandrill & 41.181 & 36.574 & 32.460 & 28.841 \\
House & 42.578 & 38.866 & 35.436 & 32.288 \\
Peppers & 41.568 & 37.778 & 35.589 & 33.752 \\
Sailboat & 41.797 & 37.309 & 33.826 & 31.421 \\
Splash & 42.703 & 39.999 & 38.058 & 36.280 \\
\hline \hline Average & 41.950 & 38.262 & 35.346 & 32.830 \\
\hline
\end{tabular}

Table 3. Coding time of proposed algorithm (Sec.)

\begin{tabular}{c||c|c|c|c}
\hline Image & QP:22 & QP:27 & QP:32 & QP:37 \\
\hline \hline Lenna & 1.600 & 1.276 & 1.119 & 0.982 \\
Mandrill & 1.965 & 1.734 & 1.498 & 1.269 \\
House & 1.864 & 1.608 & 1.406 & 1.310 \\
Peppers & 2.092 & 1.767 & 1.498 & 1.335 \\
Sailboat & 2.725 & 2.315 & 1.923 & 1.702 \\
Splash & 1.203 & 1.055 & 0.897 & 0.835 \\
\hline \hline Average & 1.909 & 1.626 & 1.391 & 1.239 \\
\hline
\end{tabular}

Table 4. PSNR of proposed algorithm (dB)

\begin{tabular}{c||c|c|c|c}
\hline Image & QP:22 & QP:27 & QP:32 & QP:37 \\
\hline \hline Lenna & 41.691 & 38.892 & 36.531 & 34.105 \\
Mandrill & 40.783 & 36.302 & 32.260 & 28.764 \\
House & 42.381 & 38.623 & 35.197 & 32.063 \\
Peppers & 41.352 & 37.618 & 35.365 & 33.417 \\
Sailboat & 41.554 & 37.079 & 33.665 & 31.212 \\
Splash & 42.469 & 39.818 & 37.783 & 35.977 \\
\hline \hline Average & 41.705 & 38.056 & 35.134 & 32.590 \\
\hline
\end{tabular}

Table 5. Time saving of proposed algorithm (\%)

\begin{tabular}{c||c|c|c|c}
\hline Image & QP:22 & QP:27 & QP:32 & QP:37 \\
\hline \hline Lenna & 61.2 & 63.1 & 62.2 & 63.1 \\
Mandrill & 64.8 & 65.2 & 64.3 & 64.4 \\
House & 56.4 & 53.7 & 56.7 & 55.3 \\
Peppers & 55.6 & 51.8 & 52.0 & 51.3 \\
Sailboat & 45.9 & 45.8 & 44.8 & 44.5 \\
Splash & 68.1 & 65.8 & 67.0 & 66.6 \\
\hline \hline Average & 58.7 & 57.6 & 57.9 & 57.6 \\
\hline
\end{tabular}

Table 6. The difference of PSNR for two methods

\begin{tabular}{c||c|c|c|c}
\hline Image & QP:22 & QP:27 & QP:32 & QP:37 \\
\hline \hline Lenna & -0.182 & -0.150 & -0.171 & -0.291 \\
Mandrill & -0.398 & -0.272 & -0.200 & -0.077 \\
House & -0.197 & -0.243 & -0.239 & -0.225 \\
Peppers & -0.216 & -0.160 & -0.224 & -0.335 \\
Sailboat & -0.243 & -0.230 & -0.161 & -0.209 \\
Splash & -0.234 & -0.181 & -0.275 & -0.303 \\
\hline \hline Average & -0.245 & -0.206 & -0.212 & -0.240 \\
\hline
\end{tabular}




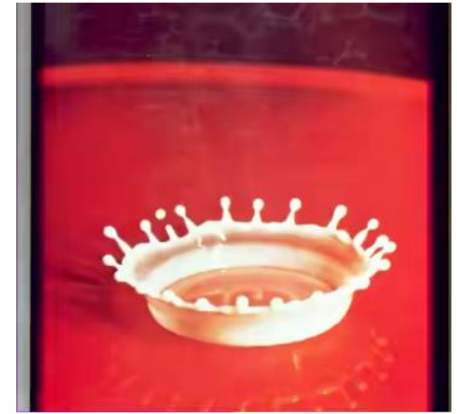

(a) Coded with HM

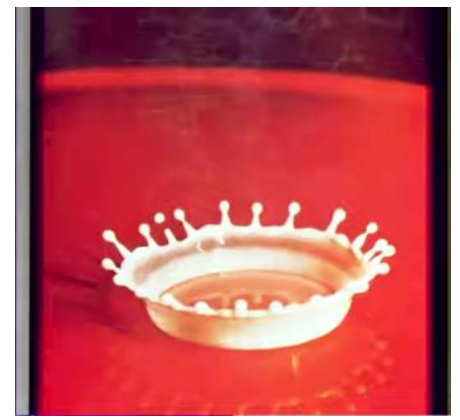

(b) Coded with proposed method

Fig. 4 Coded Splash

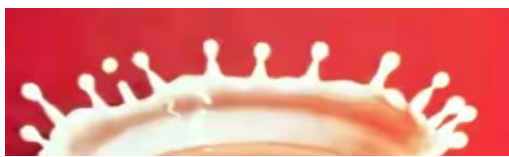

(a) Enlarged part coded with HM

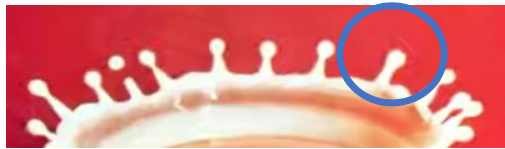

(b) Enlarged part coded with proposed

Fig. 5 Enlarged part of coded Splash, QP=37

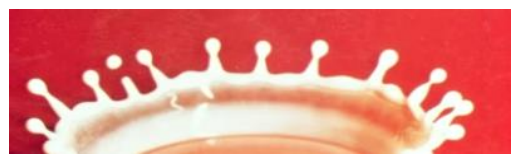

(a) Enlarged part coded with HM

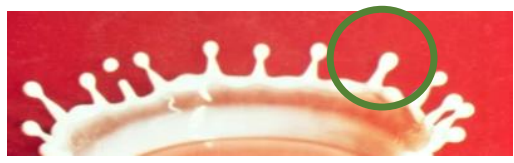

(b) Enlarged part coded with proposed

Fig. 6 Enlarged part of coded Splash, QP=22

For easy comparison, Table 5 and Table 6 show the differences in coding time (saving) and in PSNR between the two methods. Table 5 demonstrates that for all the images our

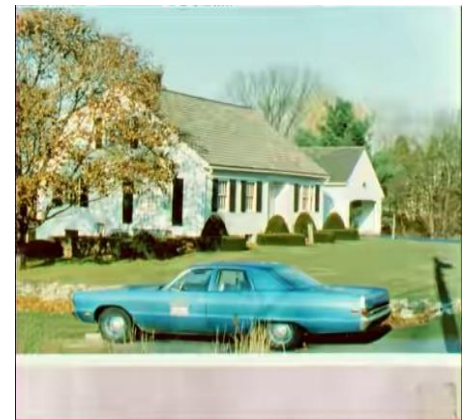

(a) Coded with HM

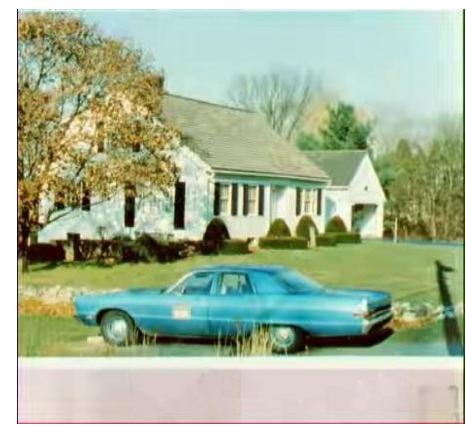

(b) Coded with proposed algorithm

Fig. 7 Coded House

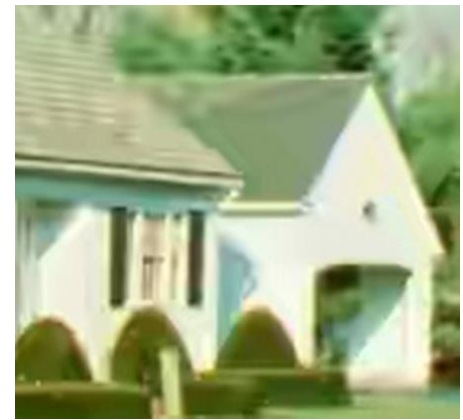

(a) Enlarged part coded with HM

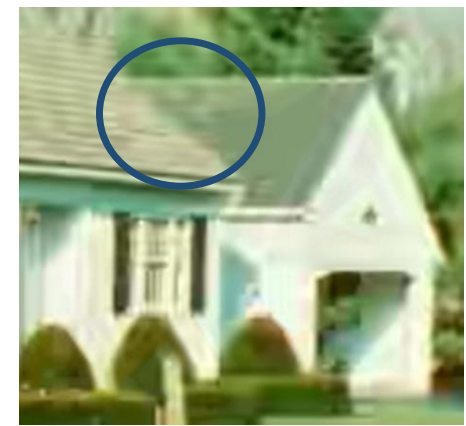

(b) Enlarged part coded with proposed algorithm

Fig. 8 Enlarged part of coded House, QP=37

algorithm saves between $44.5 \%$ (Sailboat, QP: 37 ) to $68.1 \%$ (Splash, QP: 22) in coding time, or about $58 \%$ in average. Table 6 shows that PSNR of the proposed algorithm is close 


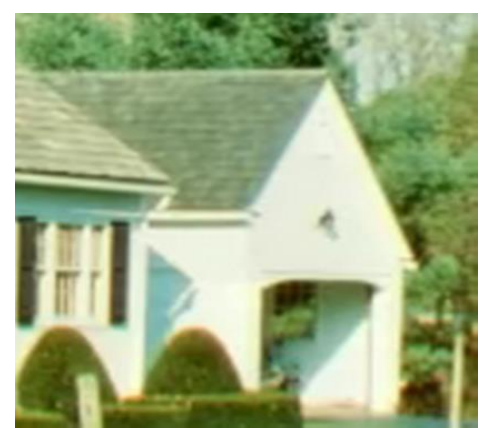

(a) Enlarged part coded with HM

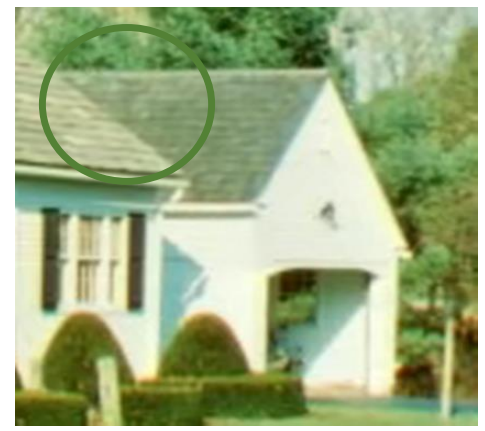

(b) Enlarged part coded with proposed algorithm

Fig. 9 Enlarged part of coded House, QP=22

to that of $\mathrm{HM}$ with an average difference of about $0.23 \%$ for all the QP selections.

To further understand the visual characteristics of the differences between the two coding methods, we compared the images coded by HM and our algorithm. Fig. 4 shows the result of Splash with $\mathrm{QP}=37$ by using HM and our algorithm. Table 5 and Table 6 demonstrate that our algorithm has a time saving of $67 \%$ at a cost of $0.30[\mathrm{~dB}]$ decrease in PSNR compared to HM. When Splash is enlarged as shown in Fig. 5, we can see that the edges of splash encoded with our algorithm are blurred (blue circle). However, for the same enlarged part encoded with $\mathrm{QP}=22$ shown in Fig 6, our algorithm gives the same clear edges (green circle) as HM. Similar trend in quality for different QP values is also observed in the House image shown in Fig. 7 to Fig. 9. The results suggest that compared to HM our algorithm provides best time saving for images encoded with larger QP at the cost of losing some minor details, but for images encoded with smaller QP the visual differences are negligible. But overall, for most commonly selected QP values our algorithm can achieve almost the same visual effects as HM.

\section{Conclusions}

In this paper we proposed a novel $\mathrm{CU}$ division algorithm to reduce computation complexity of HM while keeping coding quality similar to that of HM. Our algorithm is simple and fast and achieves similar visual effects to HM by utilizing only a few pixels around the CU. The experiment results demonstrated that our algorithm is indeed effective and can be used in HEVC intra prediction. For future improvement, one can consider taking into account the CUs for which decisions to divide have been made and the fact that motion is often spatially clustered as a way to further reduce coding time.

\section{References}

(1) JCT-VC, "WD8: Working Draft 8 of High-Efficiency Video Coding", JCTVC-J1003, JCT-VC Meeting, Stockholm, July 2012.

(2) Gary J. Sullivan, J-R Ohm, W-J. Han, and T. Wiegand, "Overview of the High Efficiency Video Coding (HEVC) Standard", IEEE Trans. Circuits and Systems for Video Tech., vol.22, no.12, pp.1649-1668, Dec. 2012.

(3) F. Bossen, B. Bross, K. Suhring, and D. Flynn, "HEVC complexity and implementation analysis" IEEE Trans. Circuits and Systems for Video Tech., vol.22, no.12, pp.1684-1695, Dec. 2012

(4) J. Lainema, F. Bossen, W-J. Han, J. Min, and K. Ugur, "Intra Coding of the HEVC standard", IEEE Trans. Circuits and Systems for Video Tech., vol.22, no.12, pp.1792-1801, Dec. 2012.

(5) I-K. K, J. Min, T. Lee, W-J. Han, and J-H. Park, "Block Partitioning Structure in the HEVC Standard", IEEE Trans. Circuits and Systems for Video Tech., vol.22, no.12, pp.1697-1706, Dec. 2012.

(6) Y. Zhang, Z. Li, and B. Li, "Gradient-based fast decision for intra prediction in HEVC", IEEE Visual Communications and Image Processing (VCIP), 2012.

(7) S. Cho and M. Kim, "Fast CU Splitting and Pruning for Suboptimal CU Partitioining in HEVC Intra Coding", IEEE Trans. Circuits and Systems for Video Tech., vol.23, no.9, pp.1555-1564, Sep. 2013.

(8) L. Shen, Z. Liu, X. Zhang, W. Zhao, and Z. Zhang, "An Effective CU Size Decision Method for HEVC Encoders", IEEE Trans. Multimedia, vol.15, no.2, Feb. 2013

(9) L. Shen, Z. Zhang, and Z. Liu, "Effective CU Size Decision for HEVC Intracoding", IEEE Trans. Image Processing, vol.23, no.10, Oct. 2014.

(10) B. Min and R. C. C. Cheung, "A Fast CU Size Decision Algorithm for the HEVC Intra Encoder", IEEE Trans. Circuits and Systems for Video Tech., vol.25, no.5, pp.892-896, May 2015. 